\title{
COMPORTAMENTO SILVICULTURAL DE ESPÉCIES FLORESTAIS EM ARRANJO PARA INTEGRAÇÃO PECUÁRIA FLORESTA
}

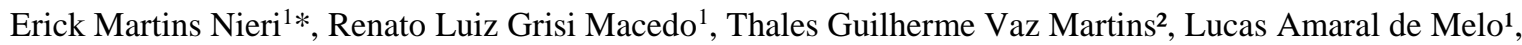 \\ Regis Pereira Venturin ${ }^{3}$, Nelson Venturin ${ }^{1}$
}

\footnotetext{
${ }^{1}$ Universidade Federal de Lavras, Programa de Pós-graduação em Engenharia Florestal, Lavras, Minas Gerais - erickenieri@ yahoo.com.br*; rlgrisi@dcf.ufla.br; lucas.amaral@dcf.ufla.br; venturin@dcf.ufla.br

${ }^{2}$ Universidade Federal de Viçosa, Programa de Pós-graduação em Ciência Florestal, Viçosa, Minas Gerais - thales.eng.florestal@gmail.com

${ }^{3}$ Empresa de Pesquisa Agropecuária de Minas Gerais, Lavras, Minas Gerais - regisventurin@ yahoo.com.br
}

\begin{abstract}
Resumo
Objetivou-se neste estudo avaliar o comportamento silvicultural de sete espécies florestais introduzidas em arranjo inicial para sistema de integração pecuária-floresta sobre pastagem degradada em Lavras, Minas Gerais. O trabalho foi instalado em delineamento de blocos casualizados em quatro repetições, 48 plantas úteis por parcela, em arranjo de $(3(3 \times 2)+7 \times 2) \mathrm{m}$. Avaliaram-se as espécies florestais Acacia mangium Willd, Calophyllum brasiliense Cambess, Dipteryx alata Vogel, Eucalyptus urophylla x Eucalyptus grandis (1528 e GG100), Khaya senegalensis A. Juss, Tectona grandis L. e dois materiais de Toona ciliata M. Roemer, propagados por meio vegetativo e via semente. As espécies foram selecionadas, principalmente, pelo valor econômico da madeira, taxa de crescimento em áreas degradadas e múltiplos usos. O comportamento silvicultural foi realizado aos 52 meses pela mensuração da sobrevivência, diâmetro à altura do peito, altura total, volume de madeira e área de projeção de copa, sendo analisados por meio do software R. Os resultados obtidos demonstram que as espécies apresentaram diferente comportamento silvicultural, destacando a adaptação e rusticidade dos híbridos de Eucalyptus urophylla x Eucalyptus grandis e Acacia mangium Willd. Contudo, dentre as espécies testadas, o híbrido de Eucalyptus urophylla $\mathrm{x}$ Eucalyptus grandis (GG100 e 1528), A. mangium e T. ciliata clonal apresentaram melhor comportamento silvicultural, com o maior potencial de utilização em sistema de integração pecuária-floresta no arranjo $(3(3 \times 2)+7 \times 2) \mathrm{m}$ em pastagem degradada, nas condições edafoclimáticas de Lavras.

Palavras-chave: silvicultura, sistema silvipastoril, introdução de espécies, adaptação.
\end{abstract}

\begin{abstract}
Silvicultural behavior of forest species in arrangement for a livestock-forestry integration. The objective of this study was to evaluate the silvicultural behavior of seven forest species introduced in initial arrangement for a livestock-forestry integration system on degraded pasture in Lavras, state of Minas Gerais, Brazil. The study was installed in a randomized block design of four replications, 48 useful plants per plot, in an arrangement of $(3(3 \times 2)+7 \times 2) \quad \mathrm{m}$. Acacia mangium Willd, Calophyllum brasiliense Cambess, Dipteryx alata Vogel, Eucalyptus urophylla $\mathrm{x}$ Eucalyptus grandis (1528 and GG100), Khaya senegalensis A. Juss, Tectona grandis L., and two materials of Toona ciliata M. Roemer, propagated by vegetative means and via seed, were assessed. The species were mainly selected for the economic value of the wood, growth rate in degraded areas, and multiple uses. Silvicultural behavior was carried out at 52 months by the measurement of survival, diameter at breast height, total height, wood volume, and crown projection area. The data were analyzed through the software $\mathrm{R}$. The results demonstrate that the species presented different silvicultural behavior, highlighting the adaptation and rusticity of the hybrids of Eucalyptus urophylla $\mathrm{x}$ Eucalyptus grandis and Acacia mangium Willd. However, among the tested species, the hybrid of Eucalyptus urophylla x Eucalyptus grandis (GG100 and 1528), A. mangium and clonal $T$. ciliata presented better silvicultural behavior and the greatest potential of use for the livestock-forestry integration system in the arrangement $(3(3 \times 2)+7 \times 2) \mathrm{m}$ in degraded pasture, under the soil and climatic conditions of Lavras.
\end{abstract}

Keywords: silviculture, silvopastoral system, introduction of species, adaptation.

\section{INTRODUÇÃO}

No Brasil, a pecuária bovina é baseada, principalmente, no pastoreio de extensas áreas. Dentre elas, 100 milhões de hectares encontram-se com algum grau de degradação. Essas áreas pouco produtivas, ou até

FLORESTA, Curitiba, PR, v. 48, n. 2, p. 195-202, abr/jun. 2018

Nieri. E. M et.al.

ISSN eletrônico 1982-4688

DOI: $10.5380 /$ rf.v48 i2.54744 
improdutivas, podem ser restauradas com a integração de componentes florestais aos animais, em arranjo adequado para a otimização da produção (DIAS FILHO, 2011).

Dentre as formas de integração, destaca-se o sistema de integração pecuária-floresta (IPF), que se caracteriza pela associação de espécies florestais com atividades pecuárias. Nesse sistema, as árvores promovem benefícios ao solo, disponibilidade de nutrientes e conforto animal, os quais permitem maior produtividade e rentabilidade ao produtor rural por meio da produção de produtos madeireiros e alimentícios (PAULA et al., 2013).

O estabelecimento adequado de um sistema de IPF é uma tarefa que exige conhecimento multidisciplinar e planejamento acurado, devendo ser considerados o desenvolvimento das culturas associadas, suas exigências ecofisiológicas e possíveis interações, além de avaliações periódicas (OLIVEIRA et al., 2009; MACEDO et al., 2010).

Por consequência, considera-se imprescindível o estudo do comportamento silvicultural das espécies inseridas em arranjo no sistema IPF, decorrente da necessidade de se obterem respostas de adaptação à região de implantação, frente às reais condições ambientais, a produtividade do material genético em estudo, de modo a estabelecer a necessidade de iluminação, e o porte alcançado de cada espécie e seu comportamento sob condições edafoclimáticas (OLIVEIRA et al., 2009; NIERI et al., 2017).

A seleção de componentes florestais e arranjos estruturados para recuperação de pastagens degradadas é uma proposição relevante e primordial para a difusão do sistema IPF no Brasil, visto a amplitude territorial e as abrangentes variações ecoclimáticas existentes. Com base nesse cenário, constata-se que a maior limitação é a inconsistência e carência de trabalhos que destaquem o potencial de espécies florestais e arranjos específicos em sistema de IPF para as diferentes regiões ecoclimáticas. Assim, nota-se a necessidade de se apresentarem espécies florestais com potencial madeireiro para serem inseridas em arranjo específico no sistema de integração pecuária floresta.

Nesse contexto, objetivou-se avaliar o comportamento silvicultural de sete espécies florestais introduzidas em arranjo inicial para sistema de integração pecuária-floresta sobre pastagem degradada aos 52 meses, em Lavras, Minas Gerais.

\section{MATERIAL E MÉTODOS}

O estudo foi desenvolvido no sul de Minas Gerais, na fazenda experimental da Empresa de Pesquisa Agropecuária de Minas Gerais (EPAMIG), unidade de Lavras, situada na latitude de $21^{\circ} 21^{\prime} 12^{\prime}$ sul e longitude de $45^{\circ} 06^{\prime}$ 51" oeste, a 890 metros acima do nível do mar. Segundo a classificação de Köppen, o clima é mesotérmico úmido do tipo $\mathrm{Cwb}$, tropical de altitude, com verões suaves. A temperatura média anual é de $19,6^{\circ} \mathrm{C}$, com precipitação média anual de $1511 \mathrm{~mm}$. A umidade relativa média anual é de $76,2 \%$ e a evaporação total anual é de 901,1 mm (ALVARES et al., 2013). O local está inserido no bioma Cerrado com pastagens, solo Latossolo Vermelho (EMBRAPA, 2013).

O condicionamento da área foi realizado no início de janeiro de 2012, uma vez que esta apresentava pastagem (Urochloa decumbens) com sintomas de degradação, conforme relatado por Dias Filho (2011). Assim, realizou-se a limpeza da área e o preparo do solo por meio de gradagem (área total) e subsolagem (linha de plantio) a 0,6 m de profundidade e a correção do solo com calcário e superfosfato simples na linha de plantio (Tabela 1).

Tabela 1. Atributos químicos do sítio experimental após correção do solo.

Table 1. Chemical attributes of the experimental site after soil correction.

\begin{tabular}{|c|c|c|c|c|c|c|c|c|c|c|c|c|c|}
\hline \multirow{2}{*}{$\begin{array}{l}\text { Prof. } \\
\text { Cm }\end{array}$} & \multirow{2}{*}{$\begin{array}{l}\mathrm{pH} \\
\mathrm{H}_{2} \mathrm{O}\end{array}$} & \multirow{2}{*}{$\begin{array}{c}\text { M.O. } \\
\text { dag } \text { kg }^{-1}\end{array}$} & $\mathbf{P}$ & $\mathbf{K}$ & $\mathbf{Z n}$ & Mn & B & \multirow[t]{2}{*}{$\mathbf{C a}$} & \multirow{2}{*}{\multicolumn{3}{|c|}{$\begin{array}{l}\text { Mg Al } \\
\text { cmol }_{\mathrm{c}} \mathbf{d m}^{-3}\end{array}$}} & $\mathbf{V}$ & $\mathbf{M}$ \\
\hline & & & \multicolumn{5}{|c|}{$\mathrm{mg} \mathrm{dm}^{-3}$} & & & & & \multicolumn{2}{|r|}{$\%$} \\
\hline $0-20$ & 5,90 & 1,64 & 7,94 & 0 & 055 & & 0,10 & 1, & 0,90 & 0 & 5,17 & 3 & \\
\hline $20-40$ & 5,70 & 1,52 & 0,56 & 40,00 & 0,13 & 4,54 & 0,13 & 1,10 & 0,50 & 0 & 4,07 & 41,83 & 0,00 \\
\hline
\end{tabular}

M.O. = Matéria Orgânica; $\mathrm{T}=$ Capacidade de Troca Catiônica a pH 7,0; $\mathrm{V}=$ Índice de saturação de bases; $\mathrm{m}=$ Índice de saturação de alumínio.

O plantio das sete espécies florestais foi realizado em fevereiro de 2012 , no arranjo $(3(3 \times 2)+7 \times 2) \mathrm{m}$, que consiste em três linhas de plantio em arranjo $3 \times 2 \mathrm{~m}$, intercaladas por uma linha simples com espaçamento de 7 x $2 \mathrm{~m}$. Esse arranjo é considerado inicial e deve ser manejado com desbastes sucessivos ao longo do tempo, visando chegar à rotação final no espaçamento de 10 x 4 m.

O delineamento experimental utilizado foi o de blocos casualizados completos em quatro repetições com parcelas de $560 \mathrm{~m}^{2}$ no arranjo $(3(3 \times 2)+7 \times 2) \mathrm{m}$, constituído de quatro linhas de plantio. Obteve-se um total de 56 plantas, das quais 48 plantas centrais foram consideradas úteis (Figura 1). Os tratamentos 
contemplaram as seguintes espécies: Acacia mangium Willd, Calophyllum brasiliense Cambess, Dipteryx alata Vogel, híbrido de Eucalyptus urophylla x Eucalyptus grandis (1528), híbrido de Eucalyptus urophylla $\mathrm{x}$ Eucalyptus grandis (GG100), Khaya senegalensis A. Juss, Tectona grandis L. F, e Toona ciliata M. Roemer, propagado por meio vegetativo e via semente.

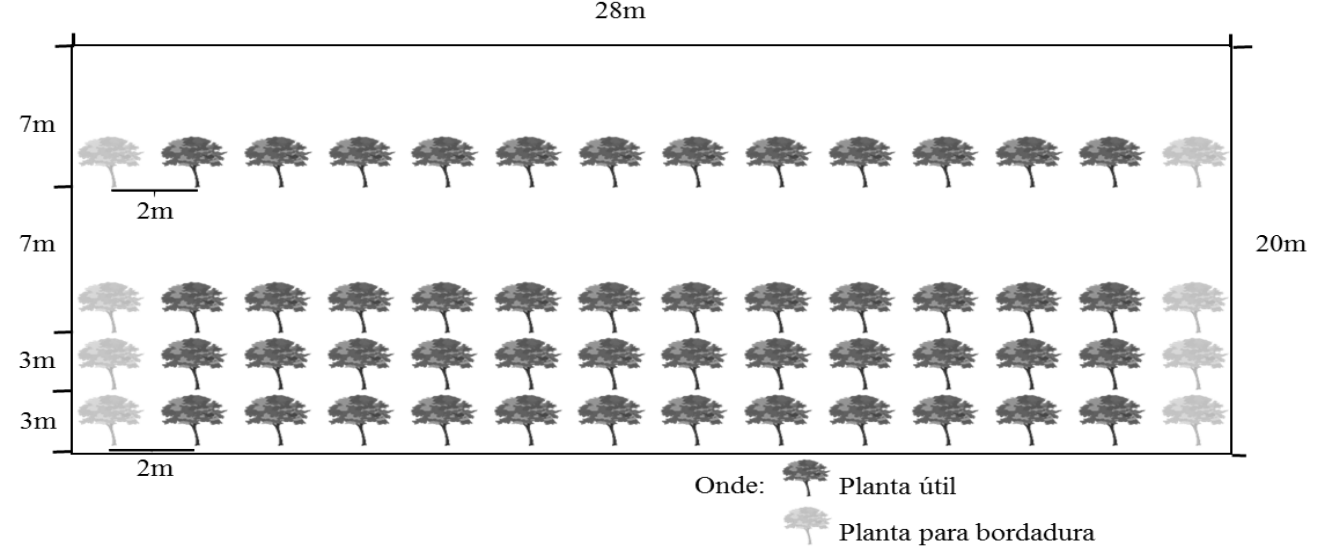

Figura 1. Representação do arranjo $(3(3 \times 2)+7 \times 2) \mathrm{m}$ e da parcela experimental para as diferentes espécies introduzidas em sistema IPF.

Figure 1. Representation of the arrangement $(3(3 \times 2)+7 \times 2) m$ and the experimental plot for the different species introduced in the IPF system.

A escolha das espécies foi fundamentada, principalmente, na disponibilidade de mudas, valor econômico da madeira (E. urophylla x E. grandis (1528 e GG100), C. brasiliense, K. senegalensis, T. grandis e T. ciliata), múltiplos usos (D. alata), fixação de nitrogênio, informações existentes na literatura e produção de bens (A. mangium).

Com 60 dias pós-plantio, foram realizados o coroamento e adubação de cobertura, com $50 \mathrm{~g}$ de NPK (20-00-20) por planta. Aos 42 meses após o plantio, efetuou-se mais uma adubação de cobertura, com a aplicação de $180 \mathrm{~g}$ de NPK (20-05-20) por planta. Além disso, realizaram-se tratos silviculturais, como o controle de formigas cortadeiras e plantas daninhas em diferentes períodos, bem como a realização de desrama (retirada de $40 \%$ da área foliar) aos 42 meses, somente para a A. mangium, a fim de reduzir o sombreamento excessivo da pastagem.

O balanço hídrico climatológico para a região de Lavras foi realizado para os o período de 2012 até julho de 2015, sendo obtidos os valores de precipitação e déficit hídrico. Aos 52 meses, foram avaliadas a sobrevivência, o diâmetro à altura do peito (DAP), a altura total das plantas (HT), o volume de madeira individual e a área de projeção de copa (APC). A sobrevivência (\%) foi obtida por meio da contagem das plantas vivas na área útil da parcela, sendo esta dividida pela quantidade total de indivíduos implantados na área útil da parcela e expressa em percentual.

O DAP e HT foram mensurados em campo por meio de suta, régua graduada e, quando necessário, hipsômetro vertex IV. O volume da madeira individual foi obtido por cubagem rigorosa, conforme metodologia de Huber. A área de projeção da copa $\left(\mathrm{m}^{2}\right)$ foi obtida por meio de medições do diâmetro da projeção da copa entre as plantas na linha de plantio (PCEP) e entre as linhas de plantio (PCEL), nos sentidos leste-oeste, norte-sul. O cálculo da área de projeção de copa foi realizado conforme Equação 1:

$$
A P C=\frac{(P C E L) \times(P C E P) \times \pi}{4}
$$

Atendidas as pressuposições estatísticas, os dados foram submetidos a análise de variância (ANOVA) e, quando se verificou significância pelo teste F, foram feitas as comparações de médias pelo teste de Scott-Knott, a 5\% de probabilidade de erro, no software estatístico R (R CORE TEAM, 2016).

\section{RESULTADOS}


Os resultados de porcentagem de sobrevivência foram transformados $(\operatorname{arcsen} \sqrt{ } \mathrm{x} / 100)$ para conferir normalidade aos dados. A sobrevivência, DAP, HT, volume de madeira por planta e APC por planta apresentaram diferenças significativas, a 5\% de probabilidade de erro, pela ANOVA (Tabela 2), sendo realizado o teste de agrupamento de médias Scott-Knott (Tabela 3).

Tabela 2. Análise de variância para sobrevivência, DAP, HT, volume e área de projeção de copa (APC) das sete espécies florestais implantadas no arranjo $(3(3 \times 2)+7 \times 2) \mathrm{m}$ em sistema de integração pecuária-floresta aos 52 meses de plantio.

Table 2. Variance analysis for survival, DAP, HT, volume, and crown projection area (APC) of seven forest species implanted in the arrangement $(3(3 \times 2)+7 \times 2) \mathrm{m}$ in a livestock-forestry integration system, at 52 months of planting.

\begin{tabular}{lcccccc}
\hline \multirow{2}{*}{$\begin{array}{l}\text { Fonte } \\
\text { de Variação }\end{array}$} & GL & $\begin{array}{c}\text { Sobrevivência } \\
(\mathbf{\%})\end{array}$ & $\begin{array}{c}\text { HT } \\
(\mathbf{m})\end{array}$ & $\begin{array}{c}\text { DAP } \\
(\mathbf{c m})\end{array}$ & $\begin{array}{c}\text { Volume } \\
\left(\mathbf{m}^{\mathbf{3}} \mathbf{p l a n t a}^{-\mathbf{1}}\right)\end{array}$ & $\begin{array}{c}\text { APC } \\
\left(\mathbf{m}^{\mathbf{2}} \mathbf{~ p l a n t a}^{-\mathbf{1}}\right)\end{array}$ \\
\hline Blocos & 3 & 169,16 & 0,43 & 1,30 & 0,000589 & 0,25 \\
Espécies & 8 & $2085,64^{* *}$ & $238,89^{* *}$ & $119,91^{* *}$ & $0,047942^{* *}$ & $62,56^{* *}$ \\
Resíduos & 24 & 82,27 & 0,96 & 0,71 & 0,000388 & 0,52 \\
\hline
\end{tabular}

Tabela 3. Médias de sobrevivência, HT, DAP, volume de madeira por planta e APC das sete espécies implantadas no arranjo $(3(3 \times 2)+7 \times 2) \mathrm{m}$ em sistema de integração pecuária-floresta, aos 52 meses de plantio.

Table 3. Means of survival, HT, DBH, wood volume, and APC of seven species implanted in the arrangement $(3(3 \times 2)+7 \times 2) \mathrm{m}$ in a livestock-forestry integration system, at 52 months of planting.

\begin{tabular}{|c|c|c|c|c|c|c|c|c|c|c|}
\hline \multirow{2}{*}{$\begin{array}{l}\text { Espécies } \\
\text { Eucalipto GG100 }\end{array}$} & \multicolumn{2}{|c|}{$\begin{array}{c}\text { Sobrevivência } \\
(\%)\end{array}$} & \multicolumn{2}{|l|}{$\begin{array}{l}\mathbf{H T} \\
(\mathbf{m})\end{array}$} & $\begin{array}{r}\text { DAP } \\
(\mathbf{c m})\end{array}$ & \multicolumn{3}{|c|}{$\begin{array}{c}\text { Volume } \\
\left(\mathrm{m}^{3} \text { planta }^{-1}\right)\end{array}$} & \multicolumn{2}{|c|}{$\begin{array}{c}\text { APC } \\
\left(\mathbf{m}^{2} \text { planta }^{-1}\right) \\
\end{array}$} \\
\hline & 86,75 & $\mathrm{a}$ & 21,56 & $\mathrm{a}$ & 16,70 & $\mathrm{a}$ & 0,3172 & $\mathrm{a}$ & 7,93 & $\bar{c}$ \\
\hline Eucalipto 1528 & 86,98 & $\mathrm{a}$ & 19,10 & $\mathrm{~b}$ & 13,00 & b & 0,1725 & $\mathrm{~b}$ & 9,37 & $\mathrm{~b}$ \\
\hline Acacia mangium & 83,33 & a & 11,27 & $\mathrm{c}$ & 12,56 & $\mathrm{~b}$ & 0,0670 & $\mathrm{c}$ & 10,93 & $\mathrm{a}$ \\
\hline Toona ciliata Clonal & 44,79 & $\mathrm{~b}$ & 4,51 & $\mathrm{~d}$ & 6,80 & $\mathrm{c}$ & 0,0126 & $\mathrm{~d}$ & 3,50 & $\mathrm{~d}$ \\
\hline Khaya senegalensis & 86,98 & a & 3,02 & $\mathrm{~d}$ & 5,04 & $\mathrm{~d}$ & 0,0114 & $\mathrm{~d}$ & 2,33 & $\mathrm{e}$ \\
\hline Tectona grandis & 93,75 & $\mathrm{a}$ & 3,09 & $\mathrm{~d}$ & 4,04 & $d$ & 0,0088 & d & 2,39 & $\mathrm{e}$ \\
\hline Toona ciliata Seminal & 42,19 & $\mathrm{~b}$ & 3,32 & $\mathrm{~d}$ & 3,79 & d & 0,0033 & d & 1,32 & $\mathrm{f}$ \\
\hline Dipteryx alata & 85,42 & $\mathrm{a}$ & 1,75 & $\mathrm{e}$ & 1,98 & $\mathrm{e}$ & 0,0013 & $\mathrm{~d}$ & 0,92 & $\mathrm{f}$ \\
\hline Calophyllum brasiliense & 41,67 & $\mathrm{~b}$ & 1,77 & $\mathrm{e}$ & 1,40 & $\mathrm{e}$ & 0,0009 & $\mathrm{~d}$ & 0,62 & $\mathrm{f}$ \\
\hline $\mathrm{CV}(\%)$ & 12,42 & & 12,69 & & 11,58 & & 29,78 & & 16,50 & \\
\hline Média & 72,43 & & 7,71 & & 7,26 & & 0,0661 & & 4,37 & \\
\hline
\end{tabular}

Médias seguidas pela mesma letra na coluna não diferem entre si pelo teste Skott-Knott, a 5\% de probabilidade de erro.

Aos 52 meses após plantio, T. grandis, eucalipto 1528, K. senegalensis, eucalipto GG100, D. alata e A. mangium apresentaram percentuais de sobrevivência superiores a 80\%. Já a baixa sobrevivência de C. brasilienses está associada, provavelmente, à baixa precipitação e ao alto déficit hídrico observado ao longo do período de estabelecimento do plantio (Figura 2).
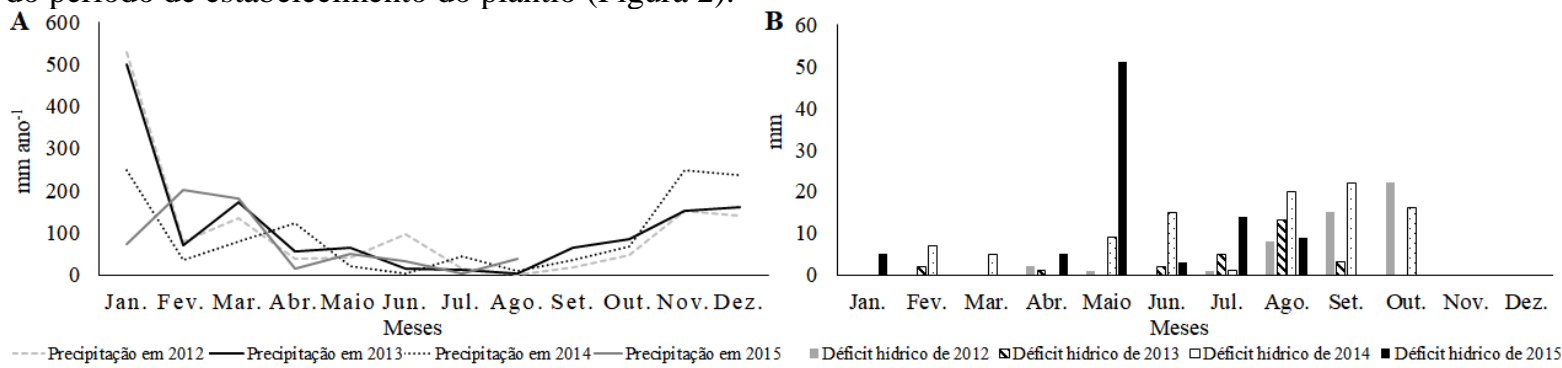

Figura 2 Precipitação média mensal e déficit hídrico em Lavras, Minas Gerais, referente aos anos de 2012, 2013, 2014 e 2015. 
Figure 2 Average monthly rainfall and water deficit in Lavras, state of Minas Gerais, Brazil, regarding the years 2012, 2013, 2014 and 2015.

Para o DAP, HT e volume por planta, observa-se que o eucalipto GG100 apresentou resultados superiores e estatisticamente diferentes em relação às demais espécies. Denota-se, assim, sua superioridade e rusticidade frente às condições edafoclimáticas da região. Em contrapartida, C. brasiliense e D. alata apresentaram o menor comportamento para DAP e HT no arranjo avaliado.

Em relação à área de projeção de copa, observa-se que A. mangium apresentou média significativamente superior comparado às demais espécies. $C$. brasiliense e D. alata apresentaram a menor área de projeção de copa. Já o eucalipto 1528 e o GG100 apresentaram resultados superiores e ideais quando comparados às demais espécies, afirmando seu potencial de serem introduzidos em sistema de integração pecuária-floresta.

\section{DISCUSSÕES}

Os percentuais de sobrevivência encontrados para T. grandes, eucalipto 1528, K. senegalensis, eucalipto GG100, D. alata e A. mangium são considerados satisfatórios. Estudos realizados por Tonini et al. (2009), Ribeiro et al. (2017), Souza et al. (2008), Pulrolnik et al. (2010) e Melotto et al. (2009) verificaram percentuais de sobrevivência similares aos do presente estudo, o que sugere a possibilidade de adaptação das espécies frente às condições edafoclimáticas da região.

Um dos parâmetros que permite constatar a real adaptação dos genótipos introduzidos em ambientes exóticos é o potencial de estabelecimento, uma vez que este expressa a capacidade de adaptação e o vigor das mudas em condições ecológicas observadas no campo após o plantio, tendo em vista a maior magnitude da interação genótipo ambiente "in loco" (MACEDO et al., 2010; NIERI et al., 2017).

Para Toona ciliata, a menor sobrevivência pode estar relacionada às condições de baixa fertilidade do solo, já que a espécie apresenta maior exigência nutricional (MORETTI et al., 2011). Sabe-se que, em áreas degradadas, as condições edáficas estão, provavelmente, entre os fatores de maior impacto na sobrevivência das plantas (CARNEVALI et al., 2016).

A menor sobrevivência de $C$. brasiliense pode estar associada à baixa disponibilidade hídrica durante a fase inicial do experimento (Figura 2), uma vez que $C$. brasiliense é uma espécie responsiva a quantidade de água disponibilizada durante o seu ciclo, com preferência em colonizar solos com alta saturação hídrica (OLIVEIRA; JOLY, 2010). A fase inicial de estabelecimento das plantas em áreas degradadas é mais crítica, pois, em condições estressantes, como períodos de seca prolongados aliados a altas temperaturas, pode reduzir a abertura estomática de forma a diminuir a perda d'água. Entretanto, também suprime a atividade fotossintética e, consequentemente, reduz as chances de sobrevivência (CARNEVALI et al., 2016).

A HT obtida neste estudo para eucalipto GG100 $(24,56 \mathrm{~m})$ foi superior à relatada para E. urophylla $\mathrm{x}$ E. grandis aos 52 meses $(19 \mathrm{~m})$, em diferentes espaçamentos no estado de São Paulo (FERREIRA et al., 2014) e para E. camaldulensis e E. pellita $(16 \mathrm{~m})$ aos 52 meses, em diferentes espaçamentos em Minas Gerais (LELES et al., 1998). Oliveira et al. (2009), ao mensurarem a HT e o DAP de um plantio de E. urophylla para sistema de integração lavoura-pecuária-floresta aos 51 meses, encontraram DAP médio de 13,58 cm e volume de $0,1218 \mathrm{~m}^{3}$. planta $^{-1}$. Ferreira et al. (2014), em reflorestamento com E. urophylla $x$ E. grandis aos 52 meses, observaram DAP médio de $13 \mathrm{~cm}$.

Ao comparar os resultados supracitados com os obtidos para ambos os eucaliptos (GG100 e 1528) neste estudo, constata-se a potencialidade dos materiais genéticos para o arranjo e ambiente testados. Isso demonstra adaptação às condições características dos sistemas de integração pecuária-floresta, assim como observado por Oliveira et al. (2015), uma vez que ambos são considerados de rápido crescimento e alta plasticidade ecofisiológica. Essa plasticidade ecofisiológica é decorrente, provavelmente, da coevolução adaptativa de seus progenitores nativos das florestas australianas chuvosas e de condições ambientais extremas e alternadas. Nesse cenário, o gênero herdou muitas adaptações propícias para seu rápido desenvolvimento em períodos favoráveis e desfavoráveis, sendo os sistemas radicular e foliar grandes precursores desse elevado desempenho (MACEDO et al., 2014).

$K$. senegalensis, $T$. grandis, $D$. alata e $C$. brasiliense apresentaram resultados inferiores aos encontrados na literatura para DAP $(5,04 \mathrm{~cm} ; 4,04 \mathrm{~cm} ; 1,98 \mathrm{~cm} ; 1,40 \mathrm{~cm}$, respectivamente $)$ e HT $(3,02 \mathrm{~m} ; 3,09 \mathrm{~m} ; 1,75 \mathrm{~m}$; $1,77 \mathrm{~m}$, respectivamente). Ribeiro et al. (2017) obtiveram valores médios de $20 \mathrm{~cm}$ para DAP e $15 \mathrm{~m}$ para HT aos 52 meses, em plantios de Khaya ivorensis em Minas Gerais. Para T. grandis, resultados superiores foram

FLORESTA, Curitiba, PR, v. 48, n. 2, p. 195-202, abr/jun. 2018.

Nieri. E. M et.al.

ISSN eletrônico 1982-4688

DOI: $10.5380 /$ rf.v48 i2.54744 
observados por Tonini et al. (2009), ao encontrarem um DAP de 5,39 m e uma HT de 8,91 m aos 48 meses, em áreas com maior precipitação média anual e menor altitude. $\mathrm{O}$ mesmo ocorreu para $D$. alata (MELOTTO et al., 2009; SOUZA et al., 2008).

Em plantios de C. brasiliense no estado de São Paulo, aos 48 meses, foram obtidos valores médios superiores ao encontrado no presente estudo para DAP $(18 \mathrm{~cm})$, HT $(3,93 \mathrm{~m})$ e volume $\left(0,01017 \mathrm{~m}^{3}\right.$.planta $\left.{ }^{-1}\right)$ (KALIL FILHO et al., 2012). Demonstra-se, assim, que a espécie não se adaptou às condições ecológicas características da região de Lavras. De acordo com a literatura e com os resultados obtidos, não se recomenda o plantio dessas espécies na região de Lavras, visto seus desenvolvimentos deficientes no sitio experimental.

O volume obtido para a A. mangium $\left(0,0670 \mathrm{~m}^{3}\right.$.planta $\left.{ }^{-1}\right)$, embora inferior ao do eucalipto (GG100 e 1528), é considerado superior ao encontrado $\left(0,03899 \mathrm{~m}^{3} \cdot\right.$ planta $\left.^{-1}\right)$ aos 52 meses para a espécie (HARDIYANTO e NAMBIAR, 2014). Esse resultado demonstra a potencialidade da espécie para ser introduzida na região de estudo.

T. ciliata clonal apresentou um desempenho satisfatório para DAP $(6,8 \mathrm{~cm})$ e HT $(4,51 \mathrm{~m})$ em comparação aos resultados para DAP $(7 \mathrm{~cm}$ e $12 \mathrm{~cm})$ e HT $(5 \mathrm{~m} \mathrm{e} 8 \mathrm{~m})$ aos 36 e 66 meses, respectivamente (CTFT, 1962). Em contrapartida, T. ciliata seminal apresentou desempenho inferior $(3,79 \mathrm{~cm}$ para DAP e 3,32 $\mathrm{m}$ para HT). Os resultados encontrados demonstram a superioridade do material genético clonado em comparação à propagação sexuada de $T$. ciliata, sendo esta indicada para a região de Lavras, assim que for controlada sua taxa de mortalidade.

A área de projeção de copa de A. mangium $\left(10,93 \mathrm{~m}^{2} \cdot\right.$ planta $\left.^{-1}\right)$ foi significativamente superior à das demais espécies estudadas. Souza et al. (2004) consideram a densa área de copa e o elevado número de ramificações por metro linear de madeira de A. mangium como características desfavoráveis para o sistema de integração pecuária floresta, pois, com o crescimento das árvores, há uma redução progressiva da luminosidade disponível para o sub-bosque, que influencia a produtividade do pasto.

Paciullo et al. (2011) avaliaram as características produtivas e nutricionais de $U$. decumbens em um sistema agrossilvipastoril com A. mangium e E. grandis. Os autores sugeriram um espaçamento ideal de 14 a $18 \mathrm{~m}$ entre as fileiras de árvores em virtude das maiores taxas de acúmulo de proteína bruta e de matéria seca, estimadas entre, aproximadamente, 7 e $9 \mathrm{~m}$ de distância de uma das fileiras. Isso indica que a distância no intervalo deve ser maior do que os sete metros adotados no presente estudo para obter maior produção de forragem.

Entretanto, vale destacar que o arranjo proposto na presente pesquisa é considerado inicial e visa facilitar o restabelecimento da pastagem ao longo do tempo. Consequentemente, visa também antecipar o seu uso por meio de um programa de desbastes sequenciais/sucessionais, em que a densidade das árvores será reduzida a 50\% aos 72 meses, por meio da retirada das colunas ímpares, transformando, assim, o espaçamento $3 \times 2 \mathrm{~m}$ em $3 \times 4 \mathrm{~m}$. Oportunamente, quando se observar competição intraespecífica no espaçamento constituído pelas três linhas de plantio $(3(3 \times 4)) \mathrm{m}$, será realizado um novo desbaste, retirando-se as 2 linhas marginais do espaçamento triplo. Após esse desbaste, permanecerá apenas a linha central de árvores (provavelmente composta por árvores com melhores conformações de fustes), com possibilidade de agregar maiores valores econômicos para explorações de madeira para serrarias.

Esse sistema possibilita a antecipação de receitas monetárias advindas dos desbastes florestais realizados e, ainda, confere agregação de valor à madeira a ser explorada e capitalização futura com sua comercialização. Além disso, o espaçamento mais adensado favorece o rápido recobrimento do solo, uma vez que, na região de estudo, prevalecem topografias íngremes associadas a solos friáveis, característicos da região de pastagem onde práticas de conservação do solo devem prevalecer prioritariamente.

\section{CONCLUSÕES}

- Dentre as espécies avaliadas, o híbrido de Eucalyptus urophylla x Eucalyptus grandis (GG100 e 1528), A. mangium e T. ciliata clonal apresentaram melhor comportamento silvicultural, com o maior potencial de utilização em sistema de integração pecuária-floresta no arranjo $(3(3 \times 2)+7 \times 2) \mathrm{m}$ em pastagem degradada nas condições edafoclimáticas de Lavras, Minas Gerais.

\section{AGRADECIMENTOS}

A CNPQ, CAPES e FAPEMIG pelo apoio financeiro para a realização desta pesquisa. 


\section{REFERÊNCIAS}

ALVARES, C.A.; STAPE, J.L.; SENTELHAS, P.C.; GONÇALVES, J.L.M.; SPAROVEK, G. Köppen's climate classification map for Brazil. Meteorologische Zeitschrift, v.22, n.6, p.711-728, 2013.

CTFT (Centre Technique Forestier Tropical). Cedrela odorata Linné et Toona ciliata M. Roemer, caractères sylvicoles et methods de plantation. Bois et Forêts des Tropiques, v.81, p.29-34, 1962.

CARNEVALI, N.H.S.; SANTIAGO, E.F.; DAlOSO, D.M.; CARNEVALI, T.O.; OLIVEIRA, M.T. Sobrevivência e crescimento inicial de espécies arbóreas nativas implantadas em pastagem degradada. Floresta, v. 46, n. 2, p. 277-286, 2016.

DIAS FILHO, M.B. Os desafios da produção animal em pastagens na fronteira agrícola brasileira. Revista Brasileira de Zootecnia, v.40, p.243-252, 2011.

EMBRAPA. Sistema brasileiro de classificação de solos. 3. ed. Brasília: Embrapa, 2013. 353p.

FERREIRA, D.H.A.A.; DOS SANTOS LELES, P.S.; MACHADO, E.C.; DE ABREU, A.H.M.; ABILIO, F.M. Crescimento de clone de Eucalyptus urophylla x E. grandis em diferentes espaçamentos. Floresta, v.44, n.3, p.431-440, 2014.

LELES, P.S.S.; REIS, M.G.F.; MORAIS, E.J. de. Relações hídricas e crescimento de árvores de Eucalyptus calmadulensis e Eucalyptus pellita sob diferentes espaçamentos na região de cerrado. Revista Árvore, v.22, n.1, p. 41-50, 1998.

HARDIYANTO, E.B.; NAMBIAR, E.K.S. Productivity of successive rotations of Acacia mangium plantations in Sumatra, Indonesia: impacts of harvest and inter-rotation site management. New Forests, v. 45, n.1, p. 557575, 2014.

KALIL FILHO, A.N.; WENDLING, I.; RIBEIRO, R.M. Seleção de guanandi em plantios comerciais. Colombo: EMBRAPA FLORESTA, 2012. 6 p.

MACEDO, R.L.G.; VALE, A.B.; VENTURIN, N. Eucalipto em sistemas agroflorestais. 1. ed. Lavras: Editora UFLA, 2010. 331p.

MACEDO, R.L.G.; VENTURIN, R.P.; VENTURIN, N.; VALE, R.S.; VALE, F.A.F. Plasticidade ecofisiológica. In: VALE, et al. Eucaliptocultura no Brasil: Silvicultura, manejo e ambiência. Viçosa: SIF, 2014. Cap. 22, p. 421-437.

MELOTTO, A.; NICODEMO, M.L.; BOCCHESE, R.A.; LAURA, C.A.; GONTIJO NETO, M.; SCHLEDER, D.D.; POTT, A.; SILVA, V.P.de. Sobrevivência e crescimento inicial em campo de espécies florestais nativas do Brasil central indicadas para sistemas silvipastoris. Revista Árvore, v.33, n.3, p.425-432, 2009.

MORETTI, B.S.; FURTINI NETO, A.E.; CARMO PINTO, S.I.D.; VOLPI FURTINI, I.; MAGALHÃES, C. A.S. Crescimento e nutrição mineral de mudas de cedro australiano (Toona ciliata) sob omissão de nutrientes. Cerne, v. 17, n. 4, 2011.

NIERI, E.M.; MACEDO, R.L.; VENTURIN, N.; VENTURIN, R.P.; JUINIOR PINTO, J.A.; MELO, L.A. Silvicultural performance of forest species introduced in integrated livestock forest system in Lavras, MG, Brazil. Ciência Rural, Santa Maria, v.47, n.12, p.1-8, 2017.

OLIVEIRA, F.L.R. de; CABACINHA, C.D.; SANTOS, L.D.T.; BARROSO, D.G.; SANTOS JUNIOR, A. dos; BRANT, M.C.; SAMPAIO, R.A. Crescimento inicial de eucalipto e acácia, em diferentes arranjos de integração lavoura-pecuária-floresta. Cerne, v. 21, n. 1, p. 227-233, 2015.

OLIVEIRA, T.K.; MACEDO, R.L.G.; VENTURIN, N.; HIGASHIKAWA, E.M. Desempenho silvicultural e produtivo sob diferentes arranjos espaciais em sistemas agroflorestais. Pesquisa Florestal Brasileira, PR, n.60, p.01-09, dez. 2009. Edição Especial. 
OLIVEIRA, V.C.; JOLY, C.A. Tolerância ao alagamento de Calophyllum brasiliense Camb.: respostas morfológicas, fisiológicas e de crescimento: (Clusiaceae). Trees, v. 24, p. 185-193, 2010.

PACIULlO, D.S.C.; GOMIDE, C. A. M.; CASTRO, C.R.T.; FERNANDES, P.B.; MÜLLER, M.D.; PIRES, M.D.F. Á.; FERNANDES, E.N; XAVIER, D. F. Características produtivas e nutricionais do pasto em sistema agrossilvipastoril, conforme a distância das árvores. Pesquisa Agropecuária Brasileira, v. 46, n. 10, p. 11761183, 2012.

PAULA, R.R.; REIS, G.G.; REIS, M.G.G.; OLIVEIRA NETO, S.N.; LEITE, H.G.; MELIDO, R.C.N.; LOPES, H.N.S.; SOUZA, F.C. Eucalypt growth in monoculture and silvopastoral systems with varied tree initial densities and spatial arrangements. Agroforestry Systems, v. 87, n. 6, p. 1295-1307, 2013.

PULROLNIK, K.; VILELA, L.; MORAES NETO, S.P. de; MARCHÃO, R.L.; GUIMARÃES JUNIOR, R. Desenvolvimento inicial de espécies arbóreas no sistema de integração lavoura-pecuária-floresta. Planaltina, DF: Embrapa Cerrados, 2010, 17p. (Boletim de pesquisa e desenvolvimento $\mathrm{n}^{\circ} 276$ )

R CORE TEAM. R: a language and environment for statistical computing. Vienna: R Foundation for Statistical Computing, 2016. Disponível em: 〈http://R-project.org>.

RIBEIRO, A., FERRAZ FILHO, A. C., SCOLFORO, J. R. S. O Cultivo do Mogno Africano (Khaya spp.) e o Crescimento da Atividade no Brasil. Revista Floresta e Ambiente, v. 24, p. 0-11, 2017.

SOUZA, C.R.; LIMA, R.M.B.; AZEVEDO, C.P.; ROSSI, L.M.B. Desempenho de espécies florestais para uso múltiplo na Amazônia. Scientia Forestalis, 36, n. 77, p. 7-14, 2008.

SOUZA, C.R.de; ROSSI, L.M.B.; AZEVEDO, C.P.de; LIMA, R.M.B.de. Comportamento da Acacia mangium e de clones de Eucalyptus grandis x E.urophylla em plantios experimentais na Amazônia Central. Scientia Forestalis, v. 1, n. 65, p. 95-101, jun. 2004.

TONINI, H.; COSTA, M.C.G.; SCHWENGBER, L.A.M. Crescimento da teca (Tectona grandis) em reflorestamento na Amazônia Setentrional. Pesquisa Florestal Brasileira, Brasilía, DF, v. 59, n. 1, p. 05-14, jul./dez. 2009. 